14-1 | 2012

Mains

\title{
Proposition d'un outil d'aide au diagnostic du syndrome du canal carpien pour les acteurs de la santé au travail
}

Proposal of a tool for diagnosis of carpal tunnel syndrome for Personal of Health at Work

Proposición de una herramienta de ayuda en el diagnóstico del síndrome del túnel carpiano para los actores de la salud en el trabajo

Florence Bazzaro et Jean-Claude Sagot

\section{(2) OpenEdition}

\section{Journals}

Édition électronique

URL : http://journals.openedition.org/pistes/737

DOI : $10.4000 /$ pistes.737

ISSN : 1481-9384

Éditeur

Les Amis de PISTES

Édition imprimée

Date de publication : 1 mai 2012

Référence électronique

Florence Bazzaro et Jean-Claude Sagot, « Proposition d'un outil d'aide au diagnostic du syndrome du canal carpien pour les acteurs de la santé au travail », Perspectives interdisciplinaires sur le travail et la santé [En ligne], 14-1 | 2012, mis en ligne le 01 mai 2012, consulté le 19 avril 2019. URL : http:// journals.openedition.org/pistes/737 ; DOI : 10.4000/pistes.737

Ce document a été généré automatiquement le 19 avril 2019

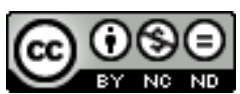

Pistes est mis à disposition selon les termes de la licence Creative Commons Attribution - Pas d'Utilisation Commerciale - Pas de Modification 4.0 International. 


\title{
Proposition d'un outil d'aide au diagnostic du syndrome du canal carpien pour les acteurs de la santé au travail
}

\author{
Proposal of a tool for diagnosis of carpal tunnel syndrome for Personal of Health \\ at Work \\ Proposición de una herramienta de ayuda en el diagnóstico del síndrome del \\ túnel carpiano para los actores de la salud en el trabajo
}

Florence Bazzaro et Jean-Claude Sagot

Nous tenons à remercier Gérard Touvenot pour sa participation à l'étude et à la conception de l'appareil cité et utilisé dans la présente communication.

\section{Introduction}

1 L'environnement socioéconomique actuel se caractérise par une compétition de plus en plus forte entre les entreprises. Ce constat implique une nécessité de réduire les délais, diminuer les coûts et augmenter la qualité des produits et des processus associés. Il en résulte souvent que les entreprises se focalisent plus sur le triptyque qualité-coût-délai et moins sur le facteur « humain » en ce qui concerne certaines fonctions du produit qu'elles peuvent concevoir. Ainsi, de nombreux produits, outils, systèmes, postes de travail faisant notre quotidien n'ont pas toujours été conçus pour répondre aux caractéristiques, besoins et attentes des utilisateurs/opérateurs (Sagot et coll., 2003 ; Sagot et Gomes, 2003). Ces difficultés d'usage peuvent vite devenir dangereuses pour la santé et la sécurité des opérateurs quand il s'agit de produits conçus pour la production industrielle, tels que des postes de travail. En effet, lorsque les capacités physiques humaines et/ou les capacités de perception et de raisonnement se trouvent sollicitées à leurs limites, il en découle souvent des incidents, des accidents, des maladies professionnelles telles que les 
troubles musculo-squelettiques (TMS). En accord avec Putz-Anderson (1988), les TMS peuvent se définir comme «l'ensemble des troubles qui résultent de l'accumulation de microtraumatismes, c'est-à-dire de blessures provoquées par des contraintes mécaniques de type répétitivité, force excessive, postures, angles articulatoires extrêmes. Ces dernières ont pour effet une hypersollicitation des articulations en termes d'amplitude et de force. Cette hypersollicitation est à l'origine de tendinopathies, de ténosynovites, de syndromes canalaires et d'atteintes musculaires ». Nous renvoyons le lecteur aux travaux de Aptel (2002; 2005) et de Cail (1996) pour des revues sur le sujet.

2 En 2010, les TMS représentent plus de $85 \%$ des maladies professionnelles (avec ou sans arrêt de travail) pour les actifs du régime général, et plus de $95 \%$ pour les salariés agricoles $^{1 .}$ Les cas de TMS constatés en 2010 ont engendré la perte de 9,7 millions de journées de travail et coûté 930 millions d'euros couverts par les cotisations des entreprises pour les actifs du régime général. Dans le secteur agricole, le coût se monte à 70 millions d'euros. Près de $50 \%$ de la totalité des TMS touchent le poignet (figure 1) ${ }^{2}$, dont $36 \%$ sont des syndromes du canal carpien (SCC), reconnus comme maladie professionnelle depuis 1982.

Sur le plan médical, le SCC regroupe l'ensemble des signes et des symptômes liés à la compression du nerf médian au niveau du canal carpien. Les signes cliniques du SCC sont bien établis. Ils apparaissent généralement à la main dominante et sont tout d'abord sensitifs, avant de devenir moteurs. Dans un premier temps, les symptômes correspondent à des acroparesthésies obligeant la personne à mobiliser sa main pour faire disparaitre les signes. Au début, ces paresthésies sont typiquement nocturnes, puis peuvent être déclenchées dans la journée par certains mouvements ou le maintien de position. Dans un deuxième stade, les fourmillements deviennent de plus en plus fréquents et intenses. Dans un troisième stade, l'engourdissement devient permanent, la sensibilité diminuant de plus en plus, l'atteinte motrice engendre que les personnes lâchent des objets pendant leur utilisation. Dans une dernière étape, l'atrophie musculaire apparaît.

Figure 1. Répartition des troubles musculo-squelettiques en fonction du locus d'atteinte

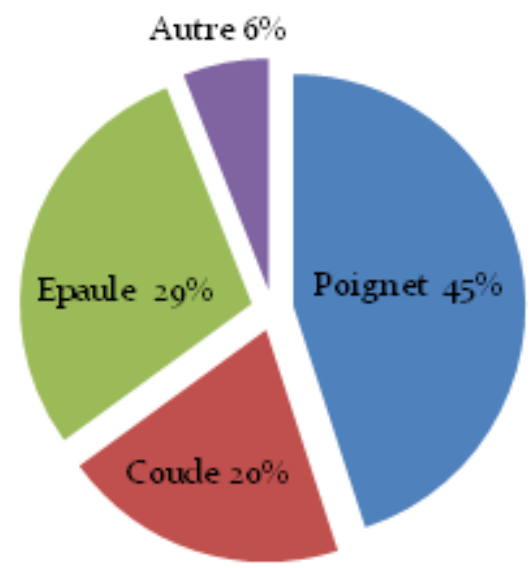

Pour réaliser le diagnostic du SCC, les acteurs de la santé au travail (médecin du travail, infirmier(e)) disposent d'un examen clinique qui consiste en un interrogatoire et des tests 
cliniques, notamment le test de Phalen et le signe de Tinel (Bates et coll., 2010). Si le syndrome est avéré, le patient est dirigé vers un neurologue spécialiste utilisant des techniques d'électrodiagnostic. Ces tests cliniques ont comme inconvénients majeurs qu'ils n'offrent pas d'informations quantitatives fiables, de critères d'interprétation avec des graduations précises, des possibilités d'enregistrement et de conservation des informations, etc.

5 À l'heure actuelle, il n'existe aucun dispositif de détection du SCC qui puisse être aisément mis en œuvre dans le cadre de la médecine du travail au même titre que les tests auditifs ou visuels pratiqués lors de la visite médicale. Ainsi, les acteurs de la santé au travail ne disposent pas d'outils évaluant et quantifiant rapidement le SCC. Consciente de cela, l'équipe d'ERgonomie et de COnception des Systèmes (ERCOS) du laboratoire Système et Transport (SeT) a développé un appareil de diagnostic rapide du SCC qui fait l'objet d'un brevet européen (Sagot et coll., 2008). Cet appareil permet de diagnostiquer en moins de cinq minutes, au moyen d'une technique simple, économique et non invasive, les risques de survenue de SCC. Il permet de faire un dépistage régulier afin de déceler, avant des atteintes graves, une diminution de la sensibilité cutanée, premier symptôme du SCC. L'objectif recherché est de doter les acteurs de la santé au travail d'un appareil fonctionnel, performant et fiable. Dans la suite de ce travail, nous décrivons succinctement l'appareil de diagnostic du SCC puis nous montrerons sa pertinence, à travers une expérimentation en milieu médical.

\section{L'appareil de diagnostic du SCC}

6 L'appareil se compose de deux éléments principaux : le corps (figure 2 (a)) et un bouton d'acquisition (figure 2 (b)). Sur la partie supérieure de l'appareil, se trouvent deux ouvertures (figure 2 (c)) : une permettant le test de la poutre (à gauche) et l'autre le test de la roue (à droite).

Figure 2. Composants de l'appareil de diagnostic du SCC

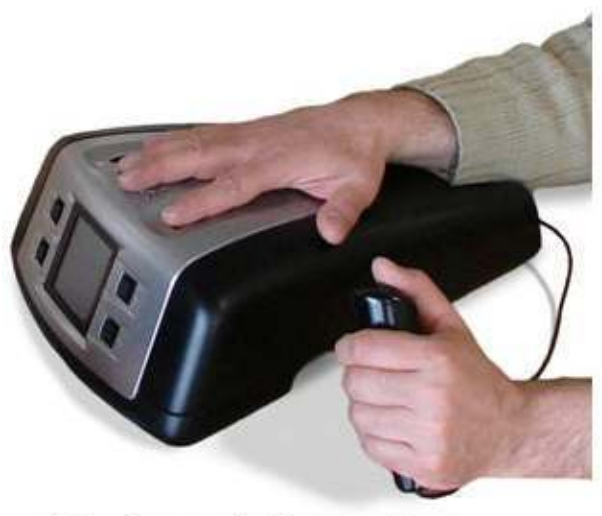

(a) - Corps de l'appareil

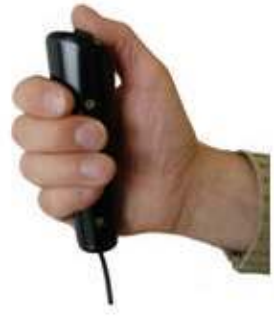

(b) - Bouton d'acquisition

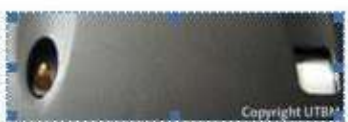

(c) - Ouvertures pour tests de la roue et de la poutre

(c) UTBM

7 Le premier test est celui de la poutre; il a pour objectif de déterminer le niveau de sensibilité à une force exercée sur le doigt. Ce test de sensibilité se différencie notamment du test des monofilaments de Semmes-Weinstein qui, pour sa part, est basé sur la 
détection de pression. Le test de la poutre se caractérise par une mesure de déformation d'un élément métallique, poutre, se déplaçant verticalement sous une charge qui varie jusqu'à ce que la personne évaluée ressente le contact d'une pastille sur son index (figure 3). La personne à évaluer pose son index (qui est un des doigts concernés, au même titre que le pouce et le majeur, par une perte de sensibilité due au SCC) sur l'ouverture du test et indique, grâce au bouton d'acquisition, à quel moment elle ressent une pression de la pastille sur son doigt. Quatre mesures successives sont effectuées: une mesure d'entraînement et trois mesures d'évaluation et une moyenne sera faite pour fournir le score global. L'expérimentateur : le médecin, l'infirmier(e) sont placés face à la personne à évaluer et à l'écran de l'appareil, ils vérifient que la personne maintient bien son bras dans l'alignement de l'ouverture, sans torsion au niveau du poignet, comme illustré sur la figure 4.

La performance mesurée est une valeur proportionnelle à la tension électrique permettant de mesurer la déformation de la jauge de contrainte installée sur la poutre. En effet, la poutre se déforme par action du doigt sur celle-ci, entrainnant la déformation de la jauge de contrainte. La performance recueillie varie de 0 à 200 , elle est proportionnelle à cette tension, elle équivaut à 0 s'il n'y a pas de déformation, à 200 pour une déformation maximale.

9 Cette performance mesurée peut être transformée en effort fourni sur le doigt. Pour une personne dite «saine ", la performance recueillie tend vers 0 , soit un effort équivalent à $0,5 \mathrm{~g}$, pour une personne souffrant de SCC, cette performance tend vers 200 , soit un effort proche de 14,3 g. À chaque démarrage, l'appareil est étalonné automatiquement. Le score est affiché sur l'écran, et peut être noté par l'expérimentateur, il est également enregistré par l'appareil.

Figure 3. Schéma du fonctionnement du test de la poutre

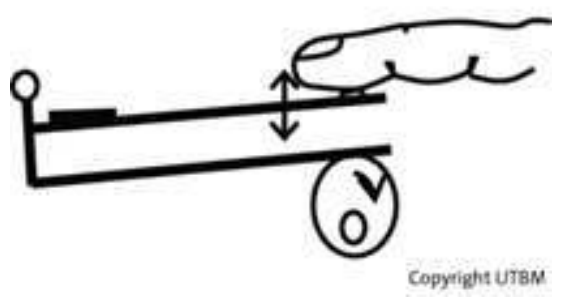


Figure 4. Positionnement du sujet (à droite) et de l'expérimentateur (à gauche) pour effectuer les tests

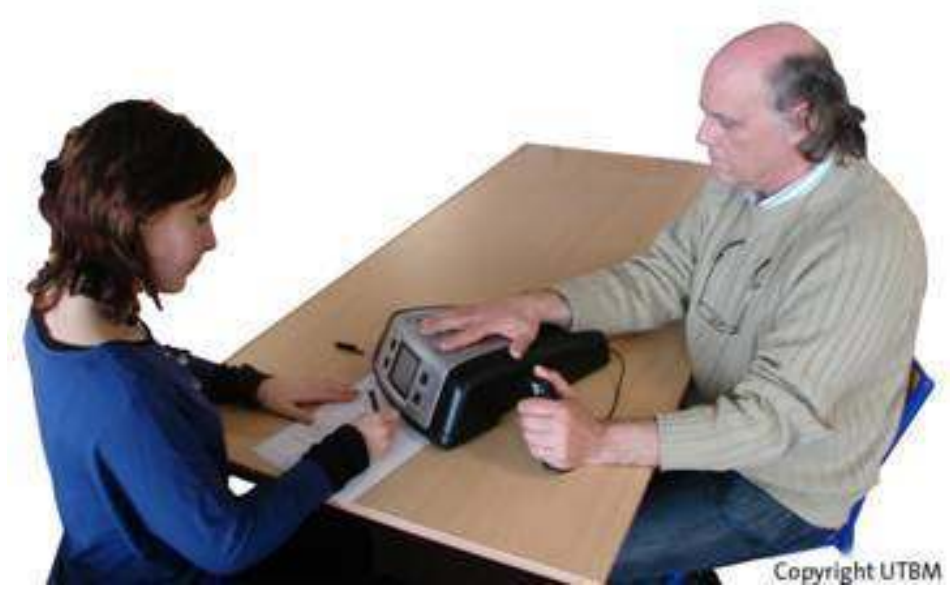

(c) UTBM.

Le test de la roue, quant à lui, vise à déterminer le niveau de sensibilité lié au pouvoir de discrimination de la personne à évaluer quant à l'importance d'une irrégularité de surface sur cette roue. Il s'agit d'une roue composée de huit encoches réparties aléatoirement, d'une largeur variant de $0,5 \mathrm{~mm}$ à $1,5 \mathrm{~mm}$ (cf. figure 5). La roue réalise plusieurs rotations et la personne indique à chaque fois qu'elle ressent une encoche au niveau de son doigt (le pouce, l'index ou le majeur selon le doigt qui est testé) grâce au bouton d'acquisition. Elle est positionnée de même que précédemment. L'élément mesuré est le nombre d'encoches détectées par le sujet. La performance est affichée mais est également enregistrée automatiquement. La performance optimale pour les personnes saines est de 24 encoches détectées. Pour une personne ayant un SCC, la performance peut tendre vers 0 encoche détectée.

Figure 5. Schéma du fonctionnement du test de la roue

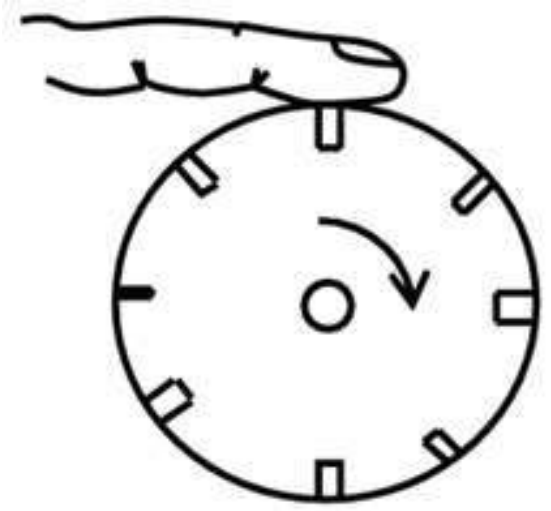

(c) UTBM

11 Afin de normaliser le protocole d'utilisation de l'appareil, il a été décidé que la poutre serait systématiquement le premier test et la roue le second.

12 Les échelles de mesure de la poutre et de la roue étant différentes pour construire un score composite, les z-scores de chacune de ces mesures ont été calculés. Ces z-scores 
permettent de normaliser les données de la population étudiée en fonction de la moyenne et de l'écart-type sur cette performance. De même, afin que l'interprétation des scores soit similaire, les performances à la poutre ont été multipliées par moins un. L'ensemble de ces opérations nous permet de calculer un score composite que nous appelons Score SCC.

Score SCC $=(\mathrm{z} \text {-score performance poutre })^{*}(-1)+(\mathrm{z}$-score performance roue $)$

\section{Méthodologie - Résultats}

Nous avons mené deux évaluations sur l'appareil en respectant le protocole d'utilisation décrit précédemment, la première avait pour vocation de valider la répétabilité des mesures effectuées. La seconde visait à traduire la pertinence de l'utilisation de cet appareil pour détecter un risque de survenue de SCC en milieu médical.

\subsection{Répétabilité des mesures}

30 sujets, étudiants ou personnels universitaires, 21 hommes et 9 femmes, ne souffrant d'aucune pathologie au niveau des doigts, poignets, coude et épaule, ayant entre 20 et 40 ans, ont testé successivement deux prototypes d'appareils en respectant le protocole défini précédemment. Ils ont réalisé les tests, une première fois le matin sur les deux appareils, et une seconde fois l'après-midi, également sur les deux appareils. Ces sujets ne ressentaient aucun des symptômes du SCC, le doigt testé était l'index. L'ordre de passage sur les deux appareils a été contrebalancé entre les sujets.

En ce qui concerne les performances à la poutre, tous les sujets, que ce soit le matin ou l'après-midi, et quel que soit l'appareil, ont obtenu le score maximal, soit un score de 0 . En ce qui concerne les performances à la roue, 28 sujets ont obtenu une performance maximale de 24. Deux sujets ont détecté 23 encoches sur les 24 , un des sujets sur l'appareil A, l'autre sujet sur l'appareil B.

ous pouvons en déduire que les performances, quel que soit l'appareil utilisé et quel que soit le moment de la journée, sont similaires. Les mesures effectuées avec les appareils de diagnostic du SCC sont répétables.

\subsection{Validation de l'appareil SCC pour un diagnostic}

17 L'objectif de cette seconde étude était de valider l'appareil de détection du SCC par comparaison avec une étude électrodiagnostique, qui est considérée comme la mesure de référence. Nous faisons l'hypothèse que l'appareil de détection du SCC permet de différencier les sujets ayant un SCC des sujets n'étant pas atteints.

La campagne d'études électrodiagnostiques a été réalisée par un neurologue. Elle a consisté en la réalisation d'un examen électrodiagnostique approfondi sur 45 sujets, 7 sujets sains ( 3 hommes, 4 femmes) et 38 sujets ( 12 hommes, 26 femmes) présentant des symptômes de SCC. Cet examen a été suivi par un diagnostic à l'aide de l'appareil de diagnostic du SCC, chaque sujet a complété le test de la poutre, puis celui de la roue avec l'index de sa main atteinte d'un SCC pour les sujets présentant des symptômes, avec l'index de sa main dominante pour les sujets sains. Les autres doigts n'ont pas été testés. 

(Dumoulin, 1980 ; Seror, 1991 ; Haloua et coll., 1994 ; Seror, 2006). Notons cependant que la littérature s'accorde généralement sur un seuil déclencheur pour un traitement chirurgical. Sur la base de la revue de Seror, en 2006, nous considérons le seuil suivant : si la vitesse sensitive est inférieure à $50 \mathrm{~m} / \mathrm{s}$ et si la latence motrice est supérieure à $4,2 \mathrm{~ms}$, alors le sujet est considéré comme étant atteint de SCC, sinon le sujet est considéré comme étant sain (tableau 1).

Tableau 1. Classification des sujets sains et des sujets atteints du SCC en fonction des résultats des études électrodiagnostiques

\begin{tabular}{|l|l|l|}
\hline & Vitesse sensitive & Latence motrice \\
\hline Sujets sains & $\geq 50 \mathrm{~m} / \mathrm{s}$ & $<4,2 \mathrm{~ms}$ \\
\hline Sujets atteints du SCC & $<50 \mathrm{~m} / \mathrm{s}$ & $\geq 4,2 \mathrm{~ms}$ \\
\hline
\end{tabular}

Après interprétation des résultats des études électrodiagnostiques, la population se définit comme suit: 7 sujets sains ( 3 hommes, 4 femmes) et 38 sujets (12 hommes, 26 femmes) atteints du SCC.

Les performances diagnostiques d'un nouveau test sont classiquement évaluées à l'aide de leur sensibilité et de leur spécificité. Nous rappelons que la sensibilité d'un test est sa capacité à indiquer qu'une personne est atteinte de la maladie quand celle-ci est présente. La spécificité, quant à elle, est la capacité d'un test à indiquer qu'une personne n'est pas atteinte d'une maladie quand celle-ci est effectivement absente. Pour compléter ces deux indicateurs, la courbe ROC (receiver operating characteristic) est fréquemment utilisée (Delacour et coll., 2005), elle offre une représentation graphique de la relation existante 
entre la sensibilité et la spécificité d'un test, calculée pour toutes les valeurs seuils possibles. La courbe ROC permet notamment de comparer les performances, en matière de diagnostic de plusieurs tests, à l'aide de l'évaluation des aires sous la courbe (ASC). L'ASC est un indice de la capacité discriminante du test et permet d'évaluer son intérêt diagnostique. Sur la base de cette ASC, il est possible de distinguer des tests pas discriminants (ASC $=0,5)$, des tests peu informatifs $(0,5 \leq$ ASC $<0,7)$, moyennement informatifs $(0,7 \leq \mathrm{ASC}<0,9)$, très informatifs $(0,9 \leq \mathrm{ASC}<1)$ et enfin des tests parfaits $($ ASC $=1)$ (Sweets, 1988). De plus, la valeur seuil optimale d'un test, permettant de discriminer les sujets sains des sujets atteints peut également être déterminée grâce à l'étude des relations entre spécificité et sensibilité. Graphiquement, cette valeur seuil correspond au point de la courbe le plus éloigné de la diagonale de référence qui représente un test d'apport nul. Ce point peut également être calculé et correspond au maximum de l'indice de Youden (Spécificité + Spécificité - 1). Pour une revue détaillée sur l'utilisation de la courbe ROC, nous renvoyons le lecteur à Delacour et coll., (2005).

Pour évaluer la sensibilité et la spécificité de notre appareil de détection du SCC en comparaison avec les études électrodiagnostiques, nous calculons trois courbes ROC, une pour le z-score calculé pour les performances à la poutre, une pour le z-score calculé pour les performances à la roue et une pour le score SCC calculé par sommation des scores précédents (cf. figure 6).

Figure 6. Courbes ROC pour le test de la poutre, de la roue et pour le score SCC en fonction du diagnostic résultant des études électrodiagnostiques

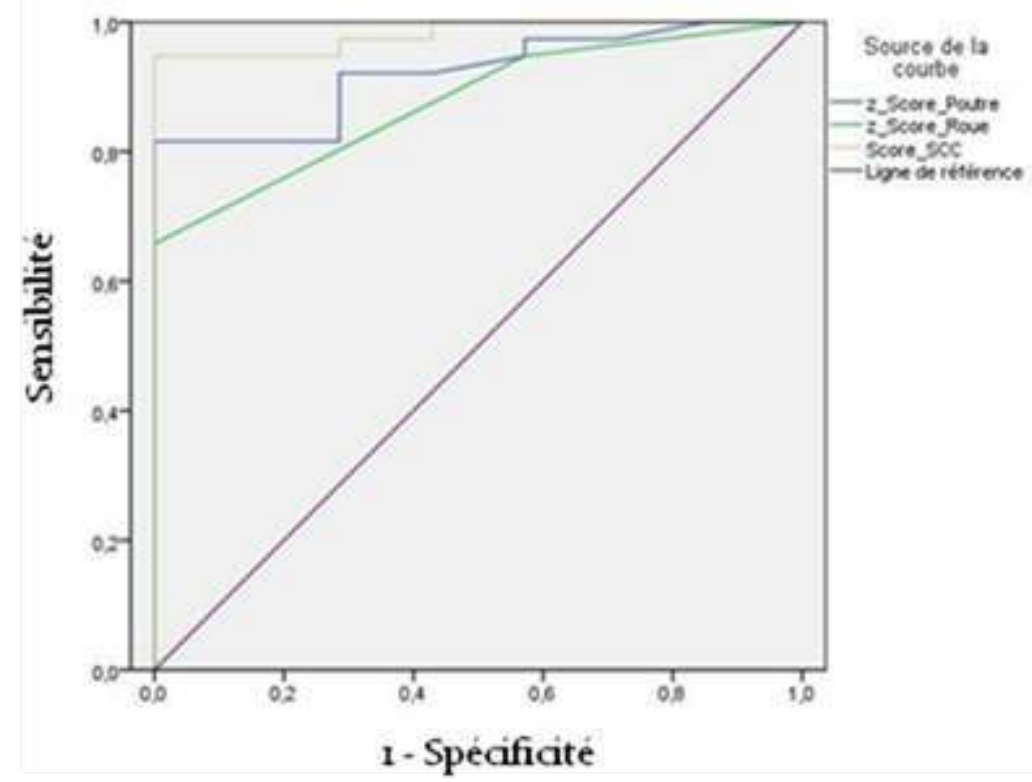

L'analyse détaillée de ces courbes ROC indique clairement que le test de la roue est considéré comme moyennement informatif et a le pouvoir discriminant le plus faible $(\mathrm{ASC}=0.876 ; \mathrm{p}<0.002)$. Le test de la poutre, quant à lui, est considéré comme très informatif (ASC $=0.921 ; p<0.000$ ), mais nous retiendrons la combinaison de ces deux tests qui apparaît comme étant également très informative, en effet la valeur de l'ASC est supérieure à celui du test de la poutre (ASC $=0.981 ; p<0.000$ ). Ces résultats montrent clairement que le Score SCC calculé à partir des performances obtenues aux tests de la roue et de la poutre a un bon pouvoir discriminant pour différencier les sujets sains des 
sujets atteints du SCC. Pour compléter notre analyse, nous avons calculé l'indice de Youden (Delacour et coll., 2005) sur l'ensemble des coordonnées de la courbe correspondant au Score SCC afin d'obtenir la valeur seuil permettant de discriminer les sujets sains des sujets atteints du SCC. Cet indice est maximum pour une valeur du Score SCC de $-1,52$. Ainsi, les sujets ayant un Score SCC inférieurs à $-1,52$ sont des sujets sains tandis que les sujets ayant un Score SCC supérieurs à $-1,52$ sont des sujets atteints du SCC.

Sujets sains $<-1,52<$ Sujets atteints du SCC

\section{Discussion}

Nous venons de présenter un nouvel appareil de diagnostic du syndrome du canal carpien (SCC). Le principal avantage de cet outil est qu'il permet d'obtenir des informations quantitatives fiables, et ce, en moins de cinq minutes, il donne un critère d'interprétation avec une graduation précise et offre des possibilités d'enregistrement et de conservation des données et des informations.

Deux résultats marquants sont à retenir. Le premier découle d'une expérimentation auprès de sujets sains, et montre que les mesures effectuées avec notre appareil de diagnostic du SCC sont répétables. Le second, pour sa part, montre la sensibilité et la spécificité de notre outil en comparaison avec une étude électrodiagnostique. Les résultats montrent que l'appareil de détection du SCC a un excellent pouvoir discriminant permettant de déceler les sujets sains et les sujets atteints du SCC, au même titre qu'un diagnostic basé sur des études électrodiagnostiques. Ces résultats indiquent également que l'utilisation complémentaire des deux tests, celui de la roue et celui de la poutre, est préférable pour parvenir à des résultats significatifs. Cette première série d'expérimentations en milieu médical nous permet donc de démontrer la pertinence de l'utilisation de cet appareil pour le du diagnostic du SCC.

De plus, il est important de noter qu'à ce jour, dans la pratique, les acteurs de la santé au travail utilisent essentiellement les tests cliniques pour diagnostiquer un SCC. Or, il n'a pas été démontré de supériorité d'une manœuvre clinique par rapport aux autres (Miedany et coll., 2008) et leur sensibilité et leur spécificité restent discutées (Fisher et coll., 2004 ; De Krom et coll., 1990). Ainsi, par exemple, une étude de Miedany et coll. (2008) conclut en démontrant que les tests de Phalen, Phalen inversé et le test de compression du nerf médian sont plus sensibles et spécifiques aux ténosynovites des fléchisseurs qu'aux SCC et que des études électrodiagnostiques complémentaires sont indispensables pour effectuer un diagnostic correct du SCC. De même, De Krom et coll. (1990) concluent que l'utilisation complémentaire de trois tests cliniques reste insuffisante pour un diagnostic correct du SCC.

Pour compléter ces tests cliniques, il est également fréquent d'utiliser des tests de sensibilité tels que le test des monofilaments, mais ces derniers ne permettent pas de contrôler certains paramètres de façon rigoureuse (Levin et coll., 1978); ainsi, les résultats peuvent être influencés par la passation, par l'expérimentateur, par son expertise dans le test (Bell-Krotoski et coll., 1993). De surcroît, ils sont extrêmement consommateurs de temps.

30 L'appareil proposé permet de répondre à l'ensemble de ces limites, en effet, son utilisation normée permet la reproductibilité des mesures, son utilisation est peu 
coûteuse en temps et il démontre une excellente sensibilité et spécificité par rapport aux études électrodiagnostiques qui restent l'indicateur déterminant du SCC (Miedany et coll., 2008, de Krom et coll., 1990).

31 Cet appareil a pour vocation d'être utilisé en milieu industriel par les acteurs de la santé au travail (médecin du travail, infirmier) et de répondre à l'absence d'outil quantitatif simple permettant à ces acteurs de diagnostiquer le SCC. La quantification du SCC, grâce à cet appareil de diagnostic du SCC, doit favoriser une meilleure compréhension de ce syndrome et particulièrement ses causes et contextes d'apparition. En effet, cette approche quantifiable du SCC permet notamment de mener des études expérimentales comparatives approfondies sur les facteurs de risque du SCC en fonction de différentes situations de travail, en fonction de différents profils de personnes, etc.

Ces premiers résultats sont encourageants, cependant ils demandent encore à être complétés. En effet, des études sont en cours pour évaluer la possibilité d'utiliser cet appareil dans un suivi longitudinal des personnes atteintes du SCC. Notre hypothèse de travail est que, pour une personne atteinte du SCC, la performance aux tests peut se dégrader au fil du temps et au fil du développement de la pathologie. En effet, les tests proposés, au contraire des tests cliniques, permettent d'évaluer quantitativement les performances des opérateurs aux tests de la roue et de la poutre au cours du temps. L'objectif est de déceler les opérateurs ayant des performances décroissantes et ainsi de pouvoir mettre en place des actions de soin ou de prévention. La détection d'un SCC peut permettre aux acteurs de la santé au travail, en collaboration avec les ergonomes et les acteurs métiers, de mener des investigations approfondies sur les différents facteurs de risques de SCC et d'ainsi améliorer rapidement les conditions de travail.

\section{BIBLIOGRAPHIE}

Aptel, M., Aublet-Cuvelier, A., Cnockaert, J.C. (2002). Les troubles musculosquelettiques du membre supérieur liés au travail. Rev Rhum, 69, 1181-1190.

Aptel, M., Aublet-Cuvelier, A. (2005). Prévenir les troubles musculo- squelettiques du membre supérieur : un enjeu social et économique. Santé publique, 17, 3, 455-469.

Aptel, M., Acheriteguy, M. (2000). Apport des kinésithérapeutes à la prévention des troubles musculosquelettiques du membre supérieur en milieu de travail. Document pour le médecin du travail, 84, 363-370.

Aptel, M. (1993). Étude dans une entreprise de montage d'appareils électroménagers des facteurs de risques professionnels du syndrome du canal carpien. Document pour le médecin du travail, 54, 149-164.

Bates, B., Bickley, L.S., Szilagyi, P.G. (2010). Guide de l'examen clinique. $6^{\mathrm{e}}$ édition, Arnette Blackwell (Eds), $1010 \mathrm{p}$.

Cail, F., Aptel, M. (1996) Les troubles musculo-squelettiques du membre supérieur. ED 797, INRS, Paris, $64 \mathrm{p}$. 
Delacour, H., Servonnet, A., Perrot, A., Vigezzi, J.F., Ramirez, J.M. (2005). La courbe ROC (receiver operating characteristic) : principes et principales applications en biologie clinique. Annales de Biologie Clinique, 63, 2, 145-154.

DeLisa, J.A. (1994). Upper extremity nerves. In, Delisa, J.A., Lee, H.J., Baron, E.M., Lai, K.S., Spieholz, N. editors, Manual of nerve conduction velocity and neurophysiology, New York, Raven Press; p. 68-71.

Dumoulin, J. (1980). Le syndrome du canal carpien et ses explorations électrophysiologiques. Electrodiagnostic-Therapie, 143-164.

Fariborz, T., Emanuel, J.T. (1993). Carpal tunnel syndrome : an ergonomics approach to its prevention. International journal of industrial ergonomics, 11, 173-179.

Haloua, J.P., Soulier, F., Colin, J.P. (1994). Potentiel sensitif de l'annulaire : Intérêt dans le diagnostic électromyographique du syndrome du canal carpien. Annales de Chirurgie de la Main, 13, 1.

Putz-Anderson, V. (1988). Cumulative Trauma disorders : a manual for musculoskeletal diseases of the upper limbs. London, Informa Healthcare.

Roquelaure, Y., Ha, C., Pélier-Cady, M.C. (2004). Réseau expérimental de surveillance épidémiologique des troubles musculo-squelettiques dans les Pays de la Loire. Surveillance en population générale du syndrome du canal carpien dans le Maine et Loire en 2002. Institut de veille sanitaire.

Rosenbaum, R.B., Ochoa, J.L. (2002). Carpal tunnel syndrome and other disorders of the median nerve. Philadelphie, Butterworth-Heunemann.

Sagot, J.C., Gomes, S., Touvenot, G. (2008). Appareil de détection du syndrome du canal carpien. Brevet européen $n^{\circ}$ 60291861 0-1265.

Sagot, J.C., Gouin, V. et Gomes, S. (2003). Ergonomics in product design: safety factor. Safety Science, 41, 137-154.

Sagot, J.C., Gomes, S. (2003). Intégration des facteurs humains dans la démarche de conception : approche ergonomique. Cahier des notes documentaires. Hygiène et sécurité au travail, 191, 61-71.

Seror, P. 1991). Examen électromyographique du membre supérieur : techniques, intérêt et limites. Dans, TUBIANA R., Traité de chirurgie de la main, Tome IV. Paris, Masson, 19-47.

Seror, P. (2006). Échographie, électroneuromyographie et syndrome du canal carpien : concurrence ou complémentarité ? Revue du rhumatisme, 73, 1324-1330.

Sweets, J.A. (1988). Measuring the accuracy of diagnosis system. Science; 240: 1285-1293.

Tanaka, S., McGlothlin, J.D. (1993). A conceptual quantitative model for prevention of workrelated carpal tunnel syndrome (CTS). International journal of industrial ergonomics, 11, 181-193.

\section{NOTES}

1. Dossier de presse Prévention des troubles musculos-quelettiques dans l'entreprise - Octobre 2011 - Ministère du Travail, de l'Emploi et de la Santé.

2. Dénombrement des maladies professionnelles déclarées et reconnues par le régime général de 2004 à 2007. Étude 2009-0051v1 - Mars 2009 - Caisse nationale de l'assurance maladie des travailleurs salariées - Direction des risques professionnels - Mission Statistique. 


\section{RÉSUMÉS}

Les acteurs de la santé au travail sont confrontés à un manque d'outils quantitatifs de suivi et de diagnostic du syndrome du canal carpien (SCC). Pour répondre à ce manque, un outil de diagnostic du SCC est proposé dans cette communication. Il est composé de deux tests : le test de la roue qui consiste à détecter des encoches sur une roue en mouvement et le test de la poutre qui détecte un niveau de sensibilité à une force exercée sur un doigt concerné par le SCC. Deux études expérimentales ont été conduites pour valider cet appareil. La première étude montre que les mesures effectuées avec l'appareil sont répétables, la seconde vise à étudier la sensibilité et la spécificité de l'outil en comparaison avec une étude électrodiagnostique. Les résultats montrent que l'appareil de détection du SCC a un excellent pouvoir discriminant permettant d'identifier les sujets sains et les sujets atteints du SCC.

People involved with occupational health at work are faced with a lack of quantitative tools to monitor and diagnose Carpal Tunnel Syndrome (CTS). In order to fill this gap, we developed a device for rapid CTS diagnosis which is composed of two complementary quantitative tests: the wheel test, which consists in detecting notches on a rotating wheel, and the beam test, which consists in detecting various forces exerted on a finger. We conducted two experimental studies to validate this device. The first one demonstrated the repeatability of the measures. The second one studied the sensitivity and specificity of our tool as compared to an electro-diagnosis test. The results show that our CTS detection device has excellent discriminatory power that allows practitioners to differentiate between healthy and CTS-affected subjects.

Los actores de la salud en el trabajo se enfrentan a una falta de herramientas cuantitativas de seguimiento y de diagnóstico del síndrome del túnel carpiano (STC). Como respuesta a esa limitante, se propone una herramienta de diagnóstico del STC compuesta de dos exámenes : el examen de la rueda, que consiste en detectar las muescas de una rueda en movimiento y el examen de la viga, que detecta un nivel de sensibilidad a una fuerza ejercida sobre un dedo afectado por el STC. Dos estudios experimentales fueron realizados para validar este dispositivo. El primero muestra que las medidas efectuadas con el equipo son repetibles; el segundo busca estudiar la sensibilidad y la especificidad de la herramienta en comparación con un estudio de electrodiagnóstico. Los resultados muestran que el dispositivo de detección del STC tiene un excelente poder discriminante, permitiendo identificar los sujetos sanos y los sujetos afectados por el STC.

\section{INDEX}

Mots-clés : syndrome du canal carpien, outil diagnostic

Palabras claves : síndrome del túnel carpiano, herramienta de diagnóstico

Keywords : carpal tunnel syndrome, diagnostic tool 


\section{AUTEURS}

\section{FLORENCE BAZZARO}

Laboratoire Systèmes et Transports (SeT - EA n 33-17), Institut de Recherche Transport-ÉnergieSociété (IRTES), Université de Technologie Belfort-Montbéliard (UTBM), 90010 Belfort Cedex

JEAN-CLAUDE SAGOT

Laboratoire Systèmes et Transports (SeT - EA n 33-17), Institut de Recherche Transport-ÉnergieSociété (IRTES), Université de Technologie Belfort-Montbéliard (UTBM), 90010 Belfort Cedex 